\title{
ErbB-2 signals through Plexin-B1 to promote breast cancer metastasis
}

\author{
Thomas Worzfeld,, ${ }^{1,2}$ Jakub M. Swiercz,, ${ }^{1,2}$ Mario Looso, ${ }^{1}$ Beate K. Straub, ${ }^{3}$ \\ Kishor K. Sivaraj, ${ }^{1}$ and Stefan Offermanns ${ }^{1,2}$
}

${ }^{1}$ Max-Planck-Institute for Heart and Lung Research, Department of Pharmacology, Bad Nauheim, Germany. ${ }^{2}$ Medical Faculty, University of Frankfurt, Frankfurt am Main, Germany. ${ }^{3}$ Institute of Pathology, University of Heidelberg, Heidelberg, Germany.

\begin{abstract}
Diagnosis of metastatic breast cancer is associated with a very poor prognosis. New therapeutic targets are urgently needed, but their development is hampered by a lack of understanding of the mechanisms leading to tumor metastasis. Exemplifying this is the fact that the approximately $30 \%$ of all breast cancers overexpressing the receptor tyrosine kinase ErbB-2 are characterized by high metastatic potential and poor prognosis, but the signaling events downstream of ErbB-2 that drive cancer cell invasion and metastasis remain incompletely understood. Here we show that overexpression of ErbB-2 in human breast cancer cell lines leads to phosphorylation and activation of the semaphorin receptor Plexin-B1. This was required for ErbB-2-dependent activation of the pro-metastatic small GTPases RhoA and RhoC and promoted invasive behavior of human breast cancer cells. In a mouse model of ErbB-2-overexpressing breast cancer, ablation of the gene encoding PlexinB1 strongly reduced the occurrence of metastases. Moreover, in human patients with ErbB-2-overexpressing breast cancer, low levels of Plexin-B1 expression correlated with good prognosis. Our data suggest that PlexinB1 represents a new candidate therapeutic target for treating patients with ErbB-2-positive breast cancer.
\end{abstract}

\section{Introduction}

Metastatic breast cancer remains essentially incurable, demonstrating the critical need for the understanding of the molecular determinants of breast cancer cell invasion and metastasis. The receptor tyrosine kinase ErbB-2 is overexpressed in about $30 \%$ of all breast cancers, and ErbB-2-overexpressing tumors are characterized by high metastatic potential and poor prognosis $(1,2)$. Several signaling proteins and pathways have been reported to contribute to the metastatic potential of ErbB-2-overexpressing breast cancers (3-5). These include transmembrane proteins such as integrins and EphA2 $(6,7)$, as well as cytosolic signaling pathways such as those mediated by Ras, Src, or the PI3K/Akt pathway (8-12). However, despite considerable progress, the signaling events mediating cancer cell invasion and metastasis in ErbB-2-overexpressing tumors are still incompletely understood, and therapeutic options for the treatment of ErbB-2-overexpressing breast cancers remain insufficient. Therefore, a better understanding of the molecular mechanisms driving ErbB-2-dependent metastasis is required to develop more effective treatments for this aggressive type of breast cancer.

Plexin-B1 belongs to a family of transmembrane receptors that mediate the cellular effects of semaphorins (13). While plexins were described in the context of axon guidance, several recent studies have established them as regulators of organogenesis, the immune system, and cancer (14-16). Plexin-B1 has been shown to interact with ErbB-2 (17). Binding of the Plexin-B1 ligand semaphorin 4D (Sema4D) to Plexin-B1 stimulates the kinase activity of ErbB-2, which leads to phosphorylation of Plexin-B1 at two specific tyrosine residues $(18,19)$. This regulation of ErbB-2 activity via Plexin-B1 and the subsequent phosphorylation of Plexin-B1 by ErbB-2 are critical for the activation of the small GTPase RhoA by the RhoGEF proteins PDZ-RhoGEF (Rho guanine nucleotide

Conflict of interest: The authors have declared that no conflict of interest exists. Citation for this article: JClin Invest. 2012;122(4):1296-1305. doi:10.1172/JCI60568. exchange factor 11) and LARG (Rho guanine nucleotide exchange factor 12), which interact with the C terminus of Plexin-B1 (18, 20-22). The Rho family of small GTPases is centrally involved in the regulation of cytoskeletal dynamics, cell adhesion, and cell migration and has been extensively studied for its role in invasion and metastasis of cancer cells (23-26). RhoA and RhoC, in particular, contribute to metastasis and poor outcome in breast cancer patients $(27,28)$.

Given that Plexin-B1 can interact with both ErbB-2 and RhoGEF proteins, Plexin-B1 may link ErbB-2 overexpression to the activation of RhoGTPases and cancer cell invasiveness. However, it is not known whether Plexin-B1 can act downstream of ErbB-2 and mediate part of its oncogenic potential. We therefore explored whether ErbB-2 overexpression leads to activation of Plexin-B1 and whether Plexin-B1 plays a role in the initiation or progression of ErbB-2-positive breast cancer.

\section{Results}

Overexpression of ErbB-2 results in activation of Plexin-B1 and Rho GTPases. To test whether overexpression of ErbB-2 is sufficient to phosphorylate and activate Plexin-B1, we overexpressed wild-type or constitutively active ErbB-2 in HEK293 cells. This resulted in tyrosine phosphorylation of Plexin-B1 (Figure 1A) as well as in activation of RhoA (Figure 1B) and RhoC (Figure 1D), but not of RhoB (Figure 1C), independent of a Plexin-B1 ligand. Expression of a Plexin-B1 mutant that lacks the intracellular domain blocked RhoA and RhoC activation, indicating that Plexin-B1 signaling is indeed required for RhoA and RhoC activation downstream of ErbB-2 (Figure 1B and Supplemental Figure 1; supplemental material available online with this article; doi:10.1172/JCI60568DS1). In addition to its ability to mediate Rho signaling, Plexin-B1 has been shown to be a GTPase-activating protein (GAP) for R-Ras (29). Consistent with earlier studies showing that R-RasGAP activity is independent of Plexin-B1 phosphorylation by ErbB-2 (18), overexpression of wild-type or 
A Plexin-B1 phosphorylation

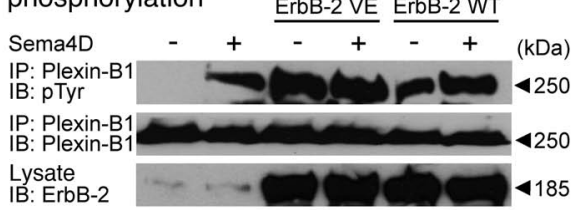

\section{RhoB activity}

Sema4D
Pull-down
IB: RhoB
Lysate
IB: RhoB
Lysate
IB: ErbB-2

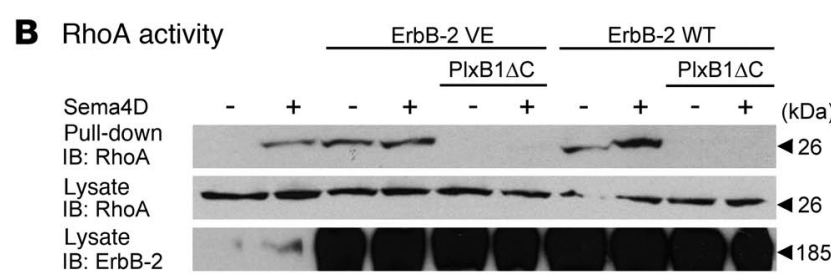

D RhoC activity

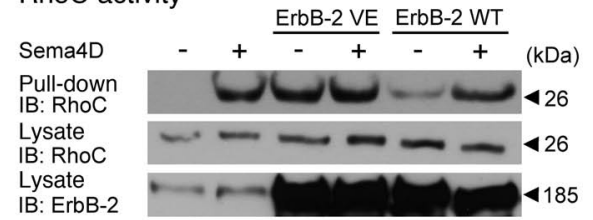

\section{E R-Ras activity}

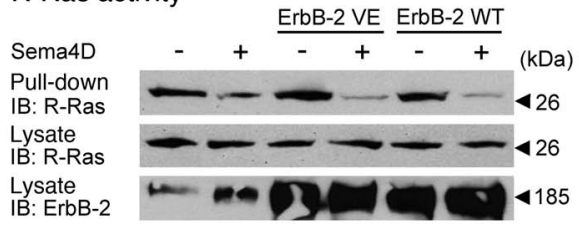

\section{Figure 1}

Overexpression of wild-type ErbB-2 or constitutively active ErbB-2 results in phosphorylation and activation of Plexin-B1. (A) HEK293 cells were transfected with VSV-Plexin-B1 and constitutively active ErbB-2 (ErbB-2 VE) or wild-type ErbB-2 (ErbB-2 WT). After incubation without $(-)$ or with (+) $150 \mathrm{nM}$ Sema4D for 20 minutes, VSV-Plexin-B1 was immunoprecipitated using an anti-VSV antibody, and precipitates were immunoblotted using anti-phosphotyrosine (pTyr) or anti-VSV antibodies. (B-E) HEK293 cells were transfected with VSV-Plexin-B1 as well as Myc-RhoA and FLAG-PDZ-RhoGEF (B), HA-RhoB and FLAG-PDZ-RhoGEF (C), HA-RhoC and FLAG-PDZ-RhoGEF (D), or HA-R-Ras and Rnd1 (E). Where indicated, cells were additionally transfected with constitutively active ErbB-2, wild-type ErbB-2, or a Plexin-B1 deletion construct that lacks the intracellular domain $(\mathrm{PlxB} 1 \Delta \mathrm{C})$. After incubation without or with $150 \mathrm{nM}$ Sema4D for 20 minutes, the indicated active Rho isoforms or R-Ras were precipitated (pull-down) as described in Methods, and precipitates were immunoblotted using antibodies directed against the tags of the Rho proteins or R-Ras.

constitutively active ErbB-2 did not affect the R-RasGAP activity of Plexin-B1 (Figure 1E). These data show that overexpression of ErbB-2 results in activation of Plexin-B1 and Rho signaling.

In buman breast cancer cells, ErbB-2 promotes invasiveness through Plexin-B1 and RhoA/RhoC. To investigate the role of ErbB-2/PlexinB1 signaling in cancer cells, we compared several human breast cancer cell lines with respect to Plexin-B1 and RhoA activity. Basal Plexin-B1 phosphorylation and RhoA activity were detectable only in cancer cell lines overexpressing ErbB-2, consistent with the notion that this pathway is active in breast cancer cells with high levels but not with low levels of ErbB-2 expression (Figure 2A). In line with this, knockdown of ErbB-2 in BT-474 cells, which endogenously express high levels of ErbB-2, resulted in a marked reduction of Plexin-B1 tyrosine phosphorylation and RhoA/RhoC activity (Figure 2B). While loss of Plexin-B1 expression in ErbB2-overexpressing BT-474 cells did not affect ErbB-2 tyrosine phosphorylation, it resulted in a strong inhibition of RhoA and RhoC activity (Figure 2, C and D). Active RhoB, which is known for its tumor suppressor function (30), could not be detected in BT-474 cells (data not shown). Thus, Plexin-B1 links ErbB-2 overexpression to the activation of RhoA and RhoC.

In light of the pro-invasive cellular effects of RhoA and RhoC, we tested whether ErbB-2 promotes tumor cell invasion by activation of RhoA and RhoC through the phosphorylation and activation of
Plexin-B1. Knockdown of Plexin-B1 had no effect on the proliferation of ErbB-2-overexpressing BT-474 cells (Figure 2E) but strongly reduced their migratory and invasive capacity (Figure $2 \mathrm{~F}$ and Supplemental Figure 2, B and C). To test whether Plexin-B1 phosphorylation by ErbB-2 was required for cancer cell invasiveness, we expressed siRNA-resistant versions of wild-type Plexin-B1 and of a Plexin-B1(Y1708F/1732F) mutant that is not phosphorylated by ErbB-2 (18). After siRNA-mediated knockdown of endogenous Plexin-B1, cells expressing phosphorylation site-deficient PlexinB1 had strongly reduced levels of active RhoA/RhoC and invaded dramatically less than cells expressing wild-type Plexin-B1 (Figure 2, $\mathrm{G}$ and $\mathrm{H}$ ). Consistent with their well-established role in cancer cell invasion, knockdown of RhoA or RhoC impaired the invasive capacity of BT-474 cells (Supplemental Figure 2D).

To further analyze the significance of the ErbB-2/Plexin-B1 receptor complex in breast cancer cells, we raised mouse monoclonal anti-Plexin-B1 antibodies and purified the extracellular domain of Plexin-B1 (PlxB1ext). Both PlxB1ext and a particular anti-Plexin-B1 antibody (clone \#93) efficiently inhibited the interaction between ErbB-2 and Plexin-B1 (Supplemental Figure 2, E-H). Uncoupling of ErbB-2 and Plexin-B1 by the anti-Plexin-B1 antibody or by PlxB1ext in BT-474 cells strongly reduced tyrosine phosphorylation of Plexin-B1 (Supplemental Figure 2, E and H), inhibited RhoA and RhoC activity (Figure 2, I and J, and Supple- 
A

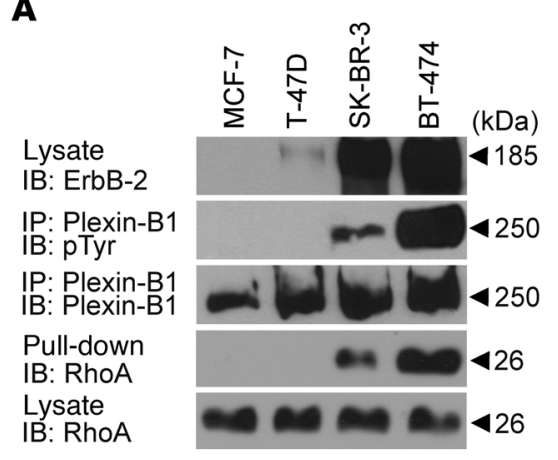

B

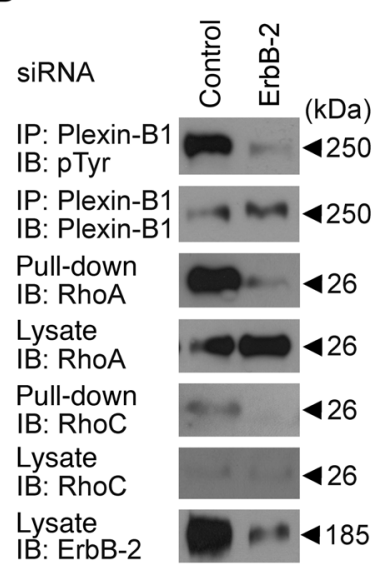

C

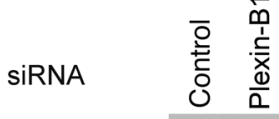

Pull-down

IB: RhoA

Lysate

IB: RhoA

Pull-down

IB: RhoC

Lysate

IB: RhoC

Lysate

IB: Plexin-B1

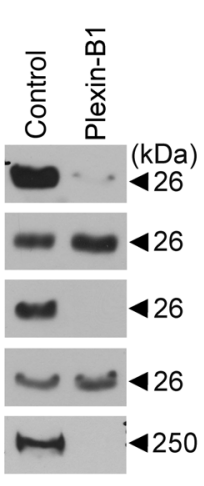

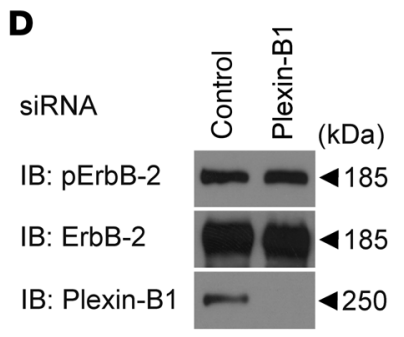

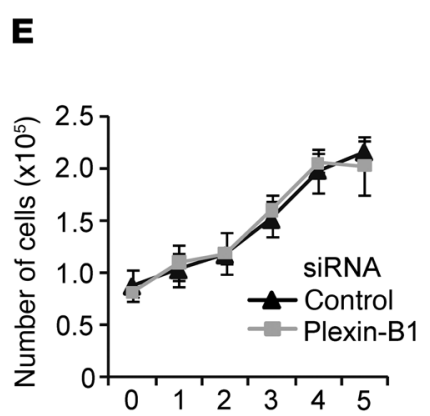

F

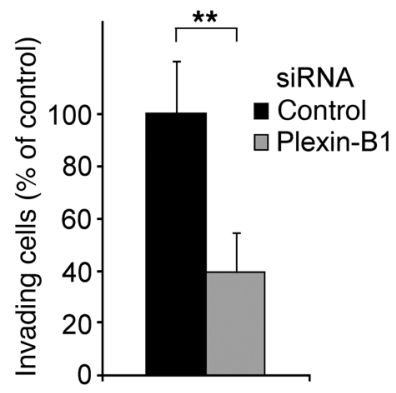

Days after siRNA transfection

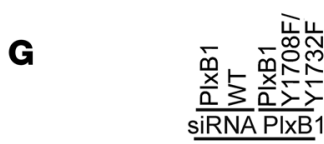

IP: Plexin-B1

IB: $p$ Tyr

IP: Plexin-B1

IB: Plexin-B1

Pull-down

IB: RhoA

Lysate

IB: RhoA

Pull-down

IB: RhoC

Lysate

IB: RhoC

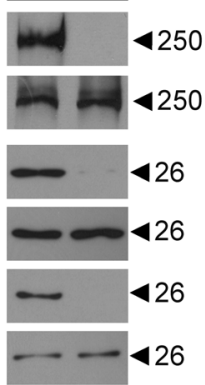

J

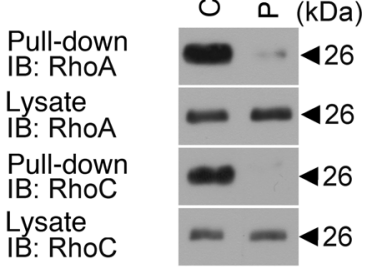

H
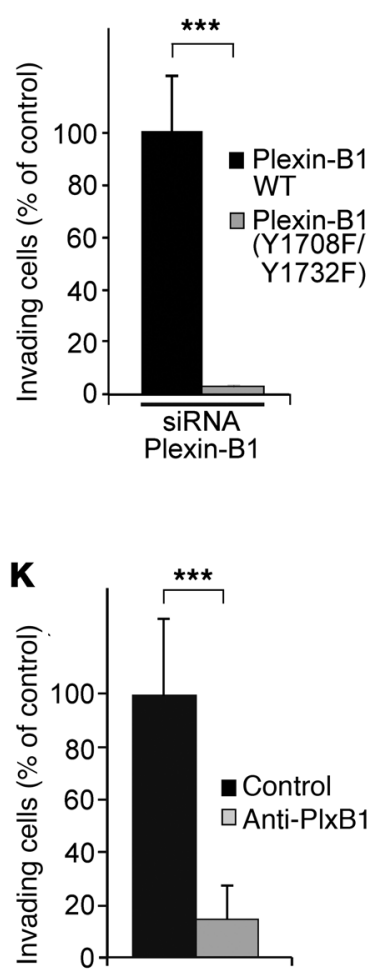

I

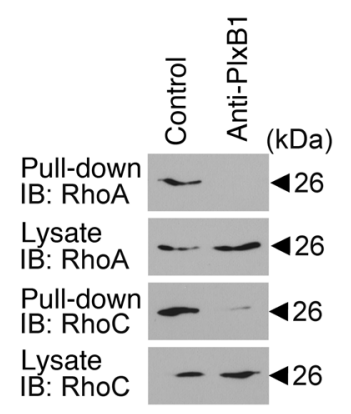




\section{Figure 2}

Plexin-B1 promotes invasion of ErbB-2-overexpressing human breast cancer cells. (A) Human breast cancer cell lines MCF-7, T-47D, SK-BR-3, or BT-474 or (B) BT-474 cells transfected with control siRNA or siRNA against ErbB-2 were lysed; Plexin-B1 was immunoprecipitated; and precipitates were immunoblotted using anti-phosphotyrosine or anti-Plexin-B1 antibodies. In a parallel experiment, levels of active RhoA/RhoC were determined. (C-F) BT-474 cells were transfected with control or Plexin-B1 siRNA. (C) The amount of Plexin-B1 and active RhoA/RhoC was determined. (D) Cell lysates were probed with an anti-phospho-ErbB-2(Y1248) antibody. (E) BT-474 cells were counted on 5 consecutive days. (F) Cells were seeded onto Matrigel-coated filters, and invading cells were counted as described in Methods. ( $\mathbf{G}$ and $\mathbf{H}$ ) BT-474 cells stably expressing siRNA-insensitive wild-type Plexin-B1 or siRNA-insensitive mutant Plexin-B1(Y1708F/Y1732F) were transfected with PlexinB1 siRNA to knock down endogenous Plexin-B1. (G) Plexin-B1 was immunoprecipitated, and precipitates were immunoblotted using anti-Plexin-B1 and anti-phosphotyrosine antibodies. In addition, levels of active RhoA/RhoC were determined. $(\mathbf{H})$ In parallel, cells were seeded onto Matrigel-coated filters, and invading cells were counted. (I and J) BT-474 cells were incubated (I) without or with a mouse monoclonal anti-Plexin-B1 antibody (anti-PIxB1; clone \#93, $1.8 \mathrm{ng} / \mu \mathrm{l})$ or $(\mathbf{J})$ without or with $150 \mathrm{nM}$ PIxB1ext, and the amounts of active RhoA/RhoC were determined. (K and $\mathbf{L}$ ) BT-474 cells were seeded onto Matrigel-coated filters in $(\mathbf{K})$ the absence or presence of a mouse monoclonal anti-Plexin-B1 antibody (anti-PIxB1; clone \#93, $1.8 \mathrm{ng} / \mu \mathrm{l})$ or $(\mathbf{L})$ the presence of $150 \mathrm{nM}$ PlxB1ext, $2 \mu \mathrm{g} / \mathrm{ml}$ trastuzumab, or both, and invading cells were counted. Data are presented as mean \pm SD. ${ }^{*} P<0.05,{ }^{* *} P<0.01,{ }^{* * *} P<0.001$.

mental Figure 2F), and decreased invasion of tumor cells (Figure $2, \mathrm{~K}$ and $\mathrm{L}$ ). The therapeutic anti-ErbB-2 antibody trastuzumab, which did not affect the interaction between ErbB-2 and PlexinB1 (data not shown), inhibited tumor cell invasion to a comparable degree as PlxB1 ext (Figure 2L), and the inhibitory effects of $\mathrm{PlxB} 1$ ext and trastuzumab on tumor cell invasion were additive (Figure 2L). In an ErbB-2-overexpressing ovarian cancer cell line, SK-OV-3, uncoupling of ErbB-2 and Plexin-B1 by the anti-PlexinB1 antibody \#93 inhibited tyrosine phosphorylation of Plexin-B1, RhoA, and RhoC activity and invasion of tumor cells (Supplemental Figure 3). Together, these data show that Plexin-B1 is required for the invasive capacity of ErbB-2-overexpressing breast and ovarian cancer cells in vitro.

In mice with ErbB-2-overexpressing breast cancer, ablation of the Plexin-B1 gene reduces metastasis. To test whether Plexin-B1 mediates ErbB-2-dependent metastasis in vivo, we employed transgenic MMTVneu mice, which overexpress wild-type ErbB-2 in mammary glands and develop metastasizing breast cancer (5). Both primary tumors and lung metastases of MMTVneu mice expressed Plexin-B1 (Supplemental Figure 4A). MMTVneu mice were crossed with Plexin-B1-deficient mice, which are viable and fertile, show normal lactation, and are devoid of any obvious defects (31-32). We found that Plexin-B1 had no effect on tumor-free survival or the size of the primary tumor (Figure 3, A and B, and Supplemental Figure 4, B and C). Examination of the histology of the primary tumors did not reveal any impact of Plexin-B1 ablation on vascularization or immune cell infiltration (Supplemental Figure 4, D-G). Consistent with the notion that Plexin-B1 signals downstream of ErbB-2, loss of Plexin-B1 expression did not affect ErbB-2 tyrosine phosphorylation of cancer cells (Supplemental Figure 4, H and I). While grading of the primary tumors was comparable in the two groups (Supplemental Figure 4, J and K), Plexin-B1-deficient tumors showed a trend toward reduced local invasiveness (Supplemental Figure 4, L and M). Examination of the lungs revealed a striking reduction in macroscopically visible metastases (Figure 3, C and D). Histological analysis confirmed a strong decrease in the number of metastases in the lung (Figure 3, E-G). These results indicate that Plexin-B1 has no effect on tumorigenesis or tumor growth but is required for metastasis of ErbB-2-dependent breast cancers in vivo.

Plexin-B1 is activated in human ErbB-2-overexpressing breast cancer, and its expression correlates with prognosis. To determine whether ErbB-2/Plexin-B1 signaling also plays a role in human breast cancer, we studied the expression of Plexin-B1 in human breast cancer tissue (Supplemental Table 1, A and B). Plexin-B1 mRNA and protein were detectable in all breast cancer tissues analyzed, independent of their ErbB-2 score (Figure 4, A and B). In samples from patients with ErbB-2-overexpressing breast cancer (Supplemental Table 1C), we found Plexin-B1 to be tyrosine phosphorylated, whereas no tyrosine phosphorylation of Plexin-B1 could be detected in ErbB-2-negative breast cancers (Figure 4C). This indicated that ErbB-2 phosphorylates and activates Plexin-B1 in human breast cancer tissue.

In microarray datasets of human breast cancer tissues, no correlation between Plexin-B1 and ErbB-2 expression levels was found (data not shown). Among patients with ErbB-2-negative breast cancer, there was a trend toward shorter disease-free survival in individuals with low expression levels of Plexin-B1 compared with those with high expression levels (Supplemental Figure 5A), which confirmed previous studies (33). In striking contrast, among patients with ErbB-2-overexpressing breast cancer, low expression levels of Plexin-B1 significantly correlated with longer disease-free survival compared with high Plexin-B1 expression levels (Figure 4D). Data on overall survival were available only for a small number of patients; however, there was a statistically non-significant trend toward higher overall survival in patients with low Plexin-B1 expression (Figure 4E). In subsets of ErbB-2-positive breast cancers stratified according to estrogen receptor (ER) status, PlexinB1 expression levels did not significantly correlate with prognosis (Supplemental Figure 5, B and C).

\section{Discussion}

Given that metastasis rather than local growth is the major prognostic factor in breast cancer, the elucidation of molecular mechanisms underlying metastasis is of crucial importance. Rho GTPases are key regulators of cell migration, and Rho signaling promotes cancer cell invasion and metastasis (23-28). The fact that Plexin-B1 can interact with ErbB-2 and also with RhoGEF proteins to regulate RhoA prompted us to test whether Plexin-B1 could act downstream of ErbB-2 and link ErbB-2 overexpression to the activation of Rho GTPases. In human breast cancer cells overexpressing ErbB-2, Plexin-B1 was tyrosine phosphorylated, and this tyrosine phosphorylation of Plexin-B1 as well as RhoA/RhoC activity were blocked by siRNA-mediated knockdown of ErbB-2. While knockdown of Plexin-B1 expression did not affect ErbB-2 tyrosine phosphorylation, it inhibited RhoA and RhoC activity as well as cancer cell invasiveness. Replacement of the endogenous Plexin-B1 protein by a mutant form of Plexin-B1 that lacks the tyrosine residues phosphorylated by ErbB-2 also strongly decreased RhoA/RhoC 
A

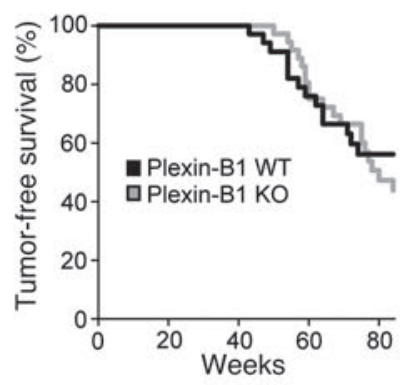

B - Plexin-B1 WT
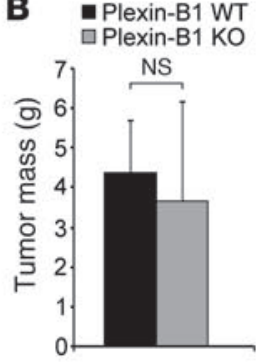

C

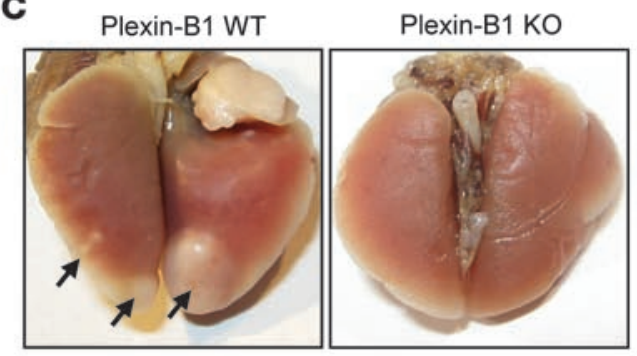

D

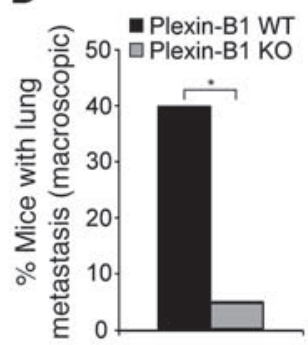

E

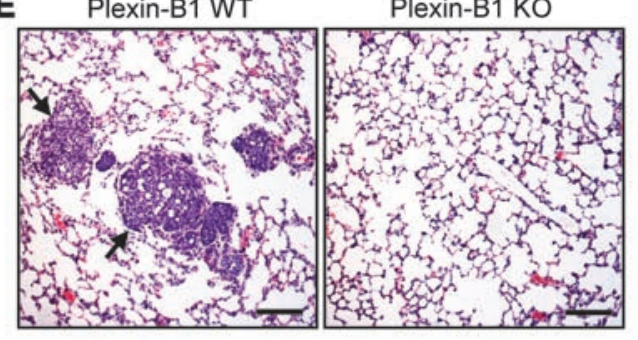

$\mathbf{F}$

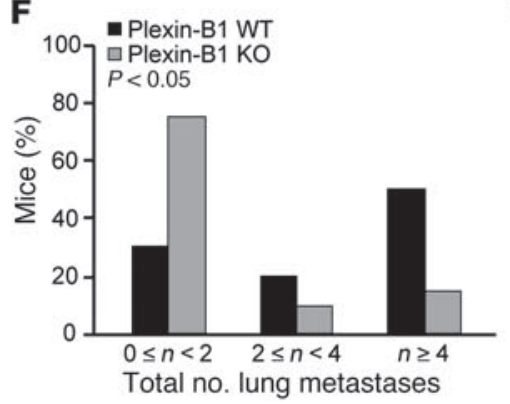

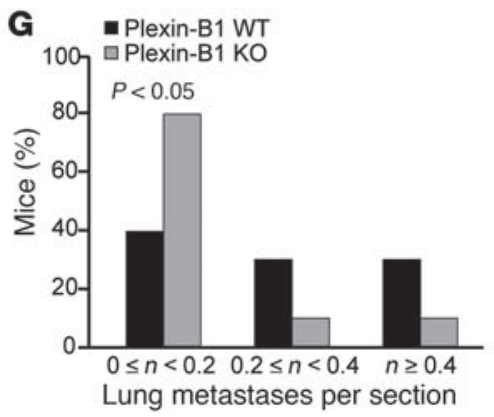

Figure 3

Plexin-B1 promotes metastasis in a mouse model of ErbB-2-overexpressing breast cancer. (A) MMTVneu;Plxnb1+/+ mice (WT) and MMTVneu; $P / x n b 1^{-/-}$mice $(\mathrm{KO})$ were examined weekly for the appearance of mammary tumors. Shown is the percentage of tumor-free survival plotted against time. WT, $n=37 ; \mathrm{KO}, n=40$. (B) Eight and one half weeks after the first appearance of a palpable tumor, mice were sacrificed, and tumors were excised and weighed. Data are presented as mean \pm SD. (C) Macroscopic images of the lungs of tumor-bearing MMTVneu;PIxnb1+/+ mice and MMTVneu;Plxnb1-/- mice. Metastases are indicated by arrows. (D) Quantification of the results in (C). (E) Microscopic images of H\&E-stained histological sections of tumor-bearing mice. Metastases are indicated by arrows. $(\mathbf{F}$ and $\mathbf{G})$ Lung sections of tumor-bearing mice were microscopically analyzed, and the metastases per lung $(\mathbf{F})$ and metastases per histological section $(\mathbf{G})$ were counted. $P$ values in $\mathbf{F}$ and $\mathbf{G}$ refer to differences between $\mathrm{WT}$ and $\mathrm{KO}$ in all groups combined. Statistical significance was determined by log-rank test $(\mathbf{A})$, $t$ test (B, F, and $\mathbf{G})$, and Fisher's exact test (D). ${ }^{*} P \leq 0.05$. Scale bars in $\mathbf{E}: 100 \mu \mathrm{m}$.

activity and the invasive capacity of cancer cells. Furthermore, interference with the interaction between ErbB-2 and Plexin-B1 by an anti-Plexin-B1 antibody or the recombinant extracellular domain of Plexin-B1 (PlxB1ext) reduced RhoA/RhoC activity and cancer cell invasion. Based on these in vitro findings, we conclude that overexpression of ErbB-2 results in phosphorylation and activation of Plexin-B1. This leads to activation of RhoGEF11/12 proteins (20-22) and RhoA/RhoC, as well as to increased cancer cell invasiveness (Figure $4 \mathrm{~F}$ ).

As in the cancer cell lines in vitro, we found that Plexin-B1 was also tyrosine phosphorylated in human breast cancer tissue overexpressing ErbB-2. In mice with ErbB-2-positive breast cancer, lack of Plexin-B1 strongly reduced metastasis, and in humans with ErbB-2-positive breast cancer, low levels of Plexin-B1 expression correlated with good prognosis. Interestingly, both knockdown of Plexin-B1 in vitro and the knockout of Plexin-B1 in vivo did not affect the proliferation of ErbB-2overexpressing cancer cells. The analysis of the histology of primary tumors in mice revealed no difference between PlexinB1-knockout and control animals with respect to vascularization or immune cell infiltration. This is in line with previous findings in xenograft cancer models (34) and argues against a major role of Plexin-B1 in cells of the tumor microenvironment. Consistent with the in vitro findings, Plexin-B1 ablation did not affect ErbB-2 tyrosine phosphorylation, which indicates that ErbB-2 is upstream of Plexin-B1. Interestingly, PlexinB1-deficient primary tumors showed a trend toward a reduction in local invasiveness, which could at least partially account for the lower rate of metastasis in these animals. We therefore conclude that Plexin-B1 is a critical downstream mediator of ErbB-2-dependent cancer cell invasion and metastasis in breast cancer. It has recently been shown that Plexin-B1 is also expressed in ovarian cancer and that knockdown of Plexin-B1 strongly inhibits the invasiveness of the ErbB-2-overexpressing ovarian cancer cell line SK-OV-3, whereas proliferation remains unchanged (35). Consistent with this, our data show that in these cells, inhibition of the interaction between ErbB-2 and Plexin-B1 by an anti-Plexin-B1 antibody strongly reduces RhoA/RhoC activity and cancer cell invasion. Therefore, it is likely that the metastasis-promoting role of Plexin-B1 is not restricted to ErbB-2-positive breast cancer but also extends to other ErbB-2-overexpressing cancers.

Conflicting data have been reported regarding the correlation of Plexin-B1 expression levels and cancer progression. While some studies have shown a downregulation of Plexin-B1 expression in melanoma and renal carcinoma, as well as a correlation between low Plexin-B1 expression levels and poor prognosis in breast carcinoma $(33,36,37)$, others have found higher Plexin-B1 expression levels in cancer tissues as compared with normal control tissues $(35,38)$. In microarray analyses we stratified the data according to 
A

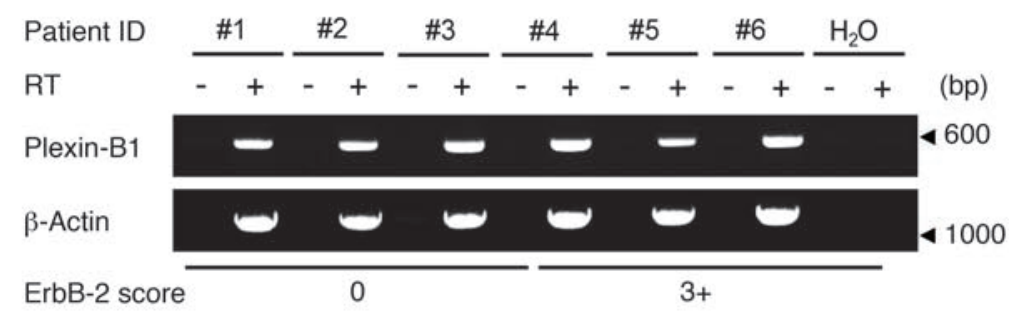

B

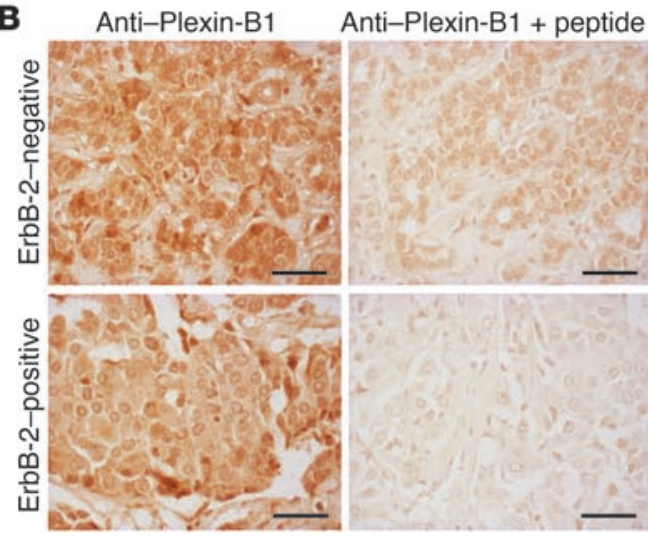

C
ErbB-2 score
IP: Plexin-B1
IB: pTyr
IP: Plexin-B1
IB: Plexin-B1
Lysate
IB: pErbB-2
Lysate
IB: ErbB-2
Lysate
IB: $\alpha$-tubulin
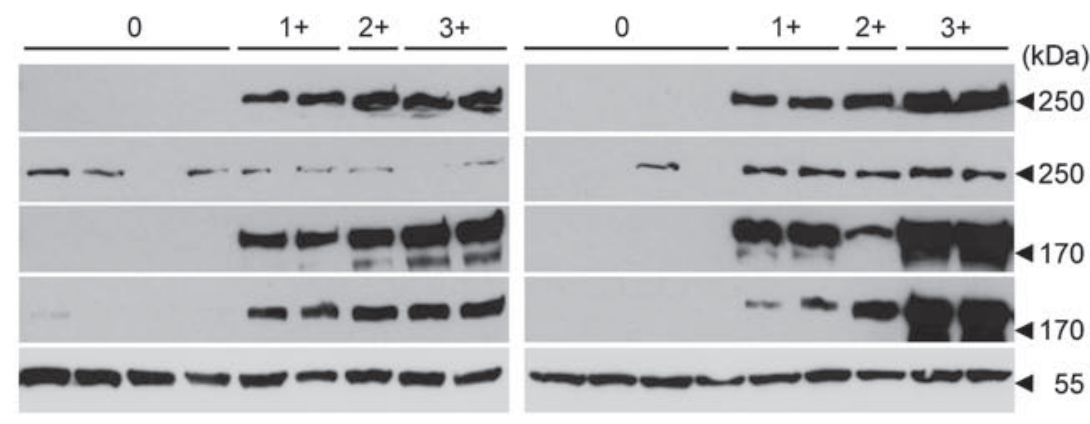

D

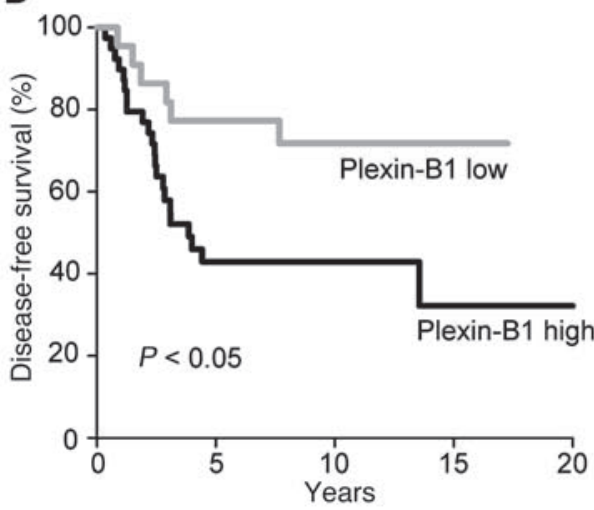

E

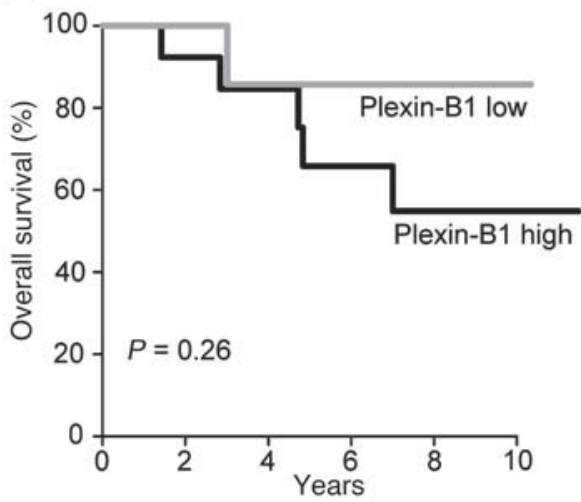

$\mathbf{F}$

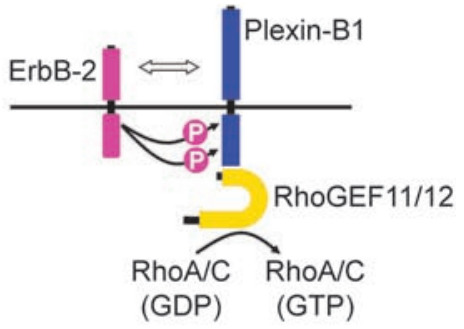

Figure 4

Plexin-B1 is activated in ErbB-2-positive human breast cancer, and its expression level correlates with prognosis of patients. (A) RNA of tumor specimens from breast cancer patients without detectable ErbB-2 expression (ErbB-2 score 0) or with ErbB-2 overexpression (ErbB-2 score $3+$ ) was isolated and reverse transcribed. PCR analysis was performed using primers specific for Plexin-B1. (B) Immunohistochemical staining of human breast cancer tissues shows that Plexin-B1 protein is expressed in cancer cells. The staining can be blocked by preincubation of the anti-Plexin-B1 antibody (R\&D Systems) with the peptide used for immunization. Scale bars: $50 \mu \mathrm{m}$. (C) Breast cancer tissues from 18 different patients without detectable ErbB-2 expression (ErbB-2 score 0) or different levels of ErbB-2 expression (ErbB-2 score 1+ to 3+) were lysed. Plexin-B1 was immunoprecipitated, and precipitates were immunoblotted using anti-phosphotyrosine or anti-Plexin-B1 antibodies. Lysates were probed for ErbB-2, phospho-ErbB-2(Y1248), and $\alpha$-tubulin. (D) Kaplan-Meier graph representing the disease-free survival of patients with ErbB-2-overexpressing breast cancer. High Plexin-B1 expression, $n=39$; low Plexin-B1 expression, $n=22$. (E) Kaplan-Meier graph representing the overall survival of patients with ErbB-2-overexpressing breast cancer. High Plexin-B1 expression, $n=13$; low Plexin-B1 expression, $n=7$. (F) Schematic illustration of the ErbB-2/Plexin-B1 signaling pathway. Overexpression of the receptor tyrosine kinase ErbB-2 results in phosphorylation of Plexin-B1 at two specific tyrosine residues. This phosphorylation of Plexin-B1 promotes the activation of RhoA and RhoC via RhoGEF 11 (PDZ-RhoGEF) and RhoGEF 12 (LARG), which stably interact with the C terminus of Plexin-B1.

expression of ErbB-2. In patients with ErbB-2-negative breast cancer, we observed a trend toward a worse prognosis when Plexin-B1 was expressed at low levels (Supplemental Figure 5A). In striking contrast, when analyzing data from patients with ErbB-2-positive breast cancer, low levels of Plexin-B1 expression significantly correlated with good prognosis. It has previously been shown that Plexin-B1 activation can induce both anti- and pro-migratory effects depending on its association with different co-receptors 
$(17,39,40)$. Thus, it is likely that the function of Plexin-B1 is context dependent and that Plexin-B1 may have different effects when coexpressed with particular sets of signaling partners in certain cancer types (41).

For patients with ErbB-2-overexpressing breast cancer, an anti-ErbB-2 antibody, trastuzumab, is approved to reduce the risk of cancer recurrence and metastasis. Trastuzumab treatment in combination with or after chemotherapy has shown significant clinical benefits (42). However, trastuzumab increases overall survival rates insufficiently, is often subject to development of resistance, and bears the risk of cardiac side effects (42-44). This underscores the need for the identification of new therapeutic targets for the treatment of ErbB-2-overexpressing breast cancers. Our data clearly indicate that the inhibition of PlexinB1 reduces cancer cell invasiveness. Under in vitro conditions we could show that also the blockade of the ErbB-2/Plexin-B1 interaction by an anti-Plexin-B1 antibody or the recombinant extracellular domain of Plexin-B1 (PlxB1ext) inhibits Rho activity and cancer cell invasiveness. In contrast, trastuzumab did not directly interfere with ErbB-2-dependent Plexin-B1 regulation. Consistent with this, the inhibitory effects of trastuzumab and $\mathrm{PlxB} 1$ ext on invasion of breast cancer cells in vitro were additive. These data indicate that the additional inhibition of Plexin-B1 signaling downstream of ErbB-2 is likely to increase the efficacy of conventional anti-ErbB-2 therapy.

The major side effect of trastuzumab is a cardiomyopathy due to the inhibition of ErbB-2 function in cardiomyocytes $(42,43)$. Similar effects can be seen in mice with cardiomyocyte-specific deletion of the Erbb2 gene, which develop dilated cardiomyopathy $(45,46)$. In contrast, Plexin-B1-deficient mice have no obvious phenotype, as loss of Plexin-B1 function is obviously compensated under physiological conditions $(31,32)$. This suggests that a therapy based on the interference with Plexin-B1-mediated signaling is less prone to side effects.

In summary, our data show that Plexin-B1 couples ErbB-2 overexpression to Rho signaling and tumor cell invasiveness and that Plexin-B1 is centrally involved in the metastasis of ErbB-2-overexpressing breast cancer. Therefore, inhibition of the ErbB-2/ Plexin-B1 interaction or of Plexin-B1-mediated signaling may reduce the risk of metastasis in patients with ErbB-2-overexpressing breast cancer and therefore represent a promising new therapeutic principle.

\section{Methods}

Antibodies. The following antibodies were used: rabbit polyclonal anti-cleaved caspase-3 (Cell Signaling Technology), rabbit polyclonal anti-CD31 (Abcam), mouse monoclonal anti-ErbB-2 (clone E2-4001, Invitrogen), rabbit polyclonal anti-phospho-ErbB-2(Y1248) (Cell Signaling Technology), rabbit polyclonal anti-phospho-ErbB-2(Y1248) (Sigma-Aldrich), rat monoclonal anti-Mac-3 (clone M3/84, BD Biosciences - Pharmingen), goat polyclonal anti-Plexin-B1 (R\&D Systems), mouse monoclonal anti-Plexin-B1 (clone 439512, R\&D Systems), rabbit monoclonal anti-RhoA (clone 67B9, Cell Signaling Technology), rabbit polyclonal anti-RhoB (Cell Signaling Technology), rabbit monoclonal anti-RhoC (clone D40E4, Cell Signaling Technology), mouse monoclonal anti- $\alpha$-tubulin (Sigma-Aldrich), goat polyclonal anti-VSV (Thermo), mouse monoclonal anti-phosphotyrosine (clone 4G10, Upstate, Millipore), mouse monoclonal anti-FLAG (clone M2, Sigma-Aldrich), rabbit polyclonal anti-Myc (Sigma-Aldrich), mouse monoclonal anti-HA (clone HA-7, Sigma-Aldrich), and trastuzumab (Genentech).
Plasmids. Eukaryotic expression plasmids carrying the human cDNAs of ErbB-2, FLAG-PDZ-RhoGEF, Myc-RhoA, HA-R-Ras, and Rnd1 were described previously (18). Human VSV-Plexin-B1 was provided by L. Tamagnone (University of Torino, Turin, Italy). HA-RhoB and HA-RhoC were obtained from D. Brandt (University of Marburg, Marburg, Germany). Human ErbB-2 V664E was provided by Axel Ullrich (Max Planck Institute of Biochemistry, Martinsried, Germany). Human VSV-Plexin-B1 $\Delta \mathrm{C}$ (PlxB1 $\Delta$ C) lacking amino acids $1514-2135$ was generated by PCR and cloned into pcDNA3.

$R N A$ extraction and RT-PCR. RNA extraction was performed using an RNeasy Kit (QIAGEN) according to the manufacturer's instructions. RTPCR was done using standard reagents and protocols (Fermentas). The following primers were used to analyze mRNA expression in human tissues: Plexin-B1 (plxnb1), 5'-CAGCCACCACTTCGTGAGTGCC-3' (sense) and 5'-GGTGACTGCCACAGCTGTTAGCTG-3' (antisense); $\beta$-actin, $5^{\prime}$-ATGGATGATGATATCGCCGCG-3' (sense) and 5'-GAAGCATTTGCGGTGGACGAT-3' (antisense). The following primers were used to analyze mRNA expression in mouse tissues: Plexin-B1 (plxnb1), 5'-GGTGGAAAGGTACTATGCAGACATCAG-3' (sense) and 5'-CCTCCTCCAGGGCAGTGATGATC-3' (antisense); $\beta$-actin, $5^{\prime}$-GGTGTGATGGTGGGAATGGGTCAG3' (sense) and 5'-GAGGAAGAGGATGCGGCAGTGG-3' (antisense). All primers were intron-spanning.

siRNAs. The sequence of the siRNA used to knockdown plexin-B1 expression was ACCACGGUCACCCGGAUUC (IBA). The control siRNA and the siRNA directed against ErbB-2 were purchased from QIAGEN.

Cell culture and transfection. MCF-7 and BT-474 cells were obtained from the German Collection of Microorganisms and Cell Cultures (DSMZ). T-47D and SK-BR-3 cells were obtained from ATCC. SK-OV-3 cells were obtained from Cell Lines Service (CLS). All cell lines were cultured according to DSMZ, ATCC, and CLS protocols. BT-474 cells were transfected with siRNAs using Lipofectamine RNAiMAX (Invitrogen) according to the manufacturer's instructions.

Protein interaction studies and Rho pull-down assays were performed 48 hours after siRNA transfection. HEK293 cells were transfected with cDNA plasmids using the calcium phosphate method.

Retroviral infections. In order to obtain siRNA-insensitive Plexin-B1, silent mutations were introduced at positions $3855(\mathrm{C} \rightarrow \mathrm{T})$ and $3858(\mathrm{G} \rightarrow \mathrm{A})$ of the coding region of the $\mathrm{CDNAs}$ encoding wild-type and mutated $(\mathrm{Y} 1708 \mathrm{~F} /$ $\mathrm{Y} 1732 \mathrm{~F})$ Plexin-B1. The resulting sequences were subcloned into the retroviral vector $\mathrm{pLNCX} 2$ (Clontech). Selection and retroviral transfection were carried out as described previously (18).

Lentiviral infections. To generate BT-474 cells with a stable knockdown of Plexin-B1, we used the Mission shRNA system (Sigma-Aldrich) according to the manufacturer's instructions. Briefly, cells were infected with lentiviruses to deliver shRNAs and a puromycin resistance gene. After selection, successful knockdown was verified by Western blotting.

Western blotting and immunoprecipitation. Western blotting was performed according to standard laboratory protocols. Immunoprecipitations were carried out in ice-cold radioimmunoprecipitation buffer $(150 \mathrm{mM} \mathrm{NaCl}$, $50 \mathrm{mM}$ Tris pH 7.4, 5 mM EDTA pH 8.0, 1\% Triton X-100, 0.1\% SDS, 0.5\% sodium deoxycholate, protease inhibitors, and $2 \mathrm{mM} \mathrm{Na}_{3} \mathrm{VO}_{4}$ ).

Production and purification of recombinant peptides and proteins. Recombinant human soluble Sema4D (residues 1-657) was purified from Chinese hamster ovary cells as described previously (18). An N-terminally His-tagged recombinant peptide, comprising amino acids 35-150 of human Plexin-B1, was expressed in E. coli and purified by metal-ion affinity chromatography using nickel agarose (GenScript). This peptide corresponds to the peptide employed by R\&D Systems for the immunization of goats to raise the anti-Plexin-B1 antibody used in this study. To produce the extracellular domain of human Plexin-B1, we cloned the cDNA sequence encoding 
amino acids 20-534 of human Plexin-B1 into the pSecTag2Hygro(A) vector. The domain was purified from the supernatant of transfected HEK293 cells by metal-ion affinity chromatography using cobalt agarose (Thermo), followed by gel filtration chromatography using a Superdex 200 10/300 GL column (Amersham).

Production of anti-Plexin-B1 antibodies. Monoclonal antibodies against the purified extracellular domain (amino acids 20-534) of human Plexin-B1 were raised in mice according to the method described by Köhler and Milstein (47). Antibodies were purified from the supernatant of hybridomas by protein A/G Sepharose affinity columns.

Determination of activated Rho and Ras. The amounts of activated cellular RhoA, RhoB, RhoC, and R-Ras were determined by precipitation with a fusion protein consisting of GST and the Rho-binding domain of Rhotekin (GST-RBD) or the Ras-binding domain of Raf1 (GST-Raf1) as described previously (18). All Rho pull-down experiments were carried out after overnight starvation in medium containing $0.5 \%$ FBS. Cells were incubated with Sema4D for 20 minutes, with the recombinant extracellular part of Plexin-B1 or trastuzumab for 45 minutes, or with a mouse monoclonal anti-Plexin-B1 antibody (anti-PlxB1; clone \#93, $1.8 \mathrm{ng} / \mu \mathrm{l}$ ) for 60 minutes prior to cell lysis.

Proliferation, migration, and invasion assays. For proliferation assays, cells were seeded in 24-well plates and transfected with siRNA. Cells were then counted using a Neubauer chamber on 5 consecutive days ( 3 wells per data point). In parallel, siRNA knockdown efficiency was monitored by Western blotting. For migration assays, $5 \times 10^{4}$ BT-474 cells stably expressing control shRNA or shRNA directed against Plexin-B1 were serum starved overnight, seeded on ThinCert filter inserts with $8.0-\mu \mathrm{m}$ pore size in 24 -well plates (Greiner Bio One), and allowed to migrate against $20 \%$ serum. Twenty-four hours later, non-migrated cells on the upper surface of the filter were removed with a cotton swab, and migrated cells were stained with toluidine blue and counted. For invasion assays, cells were serum starved 24 hours after siRNA transfection. Forty-eight hours after siRNA transfection, $1 \times 10^{5}$ BT- 474 cells were seeded into Growth Factor Reduced Matrigel Invasion Chambers with $8.0-\mu \mathrm{m}$ pore size (BD Biosciences). BT-474 cells were allowed to invade against $20 \%$ serum for 48 hours. After removal of non-invading cells on the upper surface of the filter with a cotton swab, invading cells were stained with toluidine blue and counted. siRNA knockdown efficiency was routinely assessed by Western blotting. In invasion assays using SK-OV-3 cells, $2.5 \times 10^{4}$ serum-starved cells invaded against $10 \%$ serum for 16 hours.

SRE reporter gene assays. Using the calcium phosphate method, HEK293 cells were transfected with 3DA.Luc, a reporter plasmid expressing firefly luciferase under the control of a mutant serum response element (SRE.L) which lacks a ternary complex factor binding site (48), together with plasmids encoding Plexin-B1 and PDZ-RhoGEF. Thirty-six hours after transfection, cells were serum starved for 12 hours, and the activity of firefly luciferase was measured using the OneGlo Kit (Promega) according to the manufacturer's instructions. The obtained values were normalized to cell number as determined by CellTiter Fluo kit (Promega).

Sema4D binding assay. MCF-7 cells were treated without or with the mouse monoclonal anti-Plexin-B1 antibody (clone \#93). After 1 hour cells were washed with PBS and incubated with Myc-Sema4D for 30 minutes. Unbound Sema4D was removed by washing with PBS, and bound Sema4D was detected using an HRP-conjugated anti-Myc antibody. HRP activity was measured using OPD chromogenic substrate (Dako) according to the manufacturer's protocol.

Genetically altered mice. MMTVneu mice (5) were purchased from The Jackson Laboratory (stock number 002376). Plexin-B1-knockout $($ Plxnb1-/-) mice were generated as described previously (31). Female ani- mals from Plxnb1 $1^{+-} \times$MMTVneu;Plxnb1 $1^{+/}$crosses were kept as virgins for the entire period of the study. We monitored mice for tumors by palpation weekly. Mice were sacrificed 8.5 weeks after the first appearance of a palpable tumor. Tumors were excised and weighed. Pictures of the lungs were taken after fixation in $4 \%$ PFA overnight $\left(4^{\circ} \mathrm{C}\right)$ and dehydration in ethanol. Lungs were then further processed for histology and sectioned on a microtome (section thickness, $5 \mu \mathrm{m}$ ). Sections were stained with $\mathrm{H} \& \mathrm{E}$ and analyzed for the presence of metastases. The distance between the analyzed sections was $50 \mu \mathrm{m}$.

Histology and immunobistochemistry. The grading of MMTVneu primary tumors was scored on H\&E-stained sections. For every tumor analyzed, a sub-score $(1,2$, or 3$)$ was assigned to each of the following parameters: tubule formation (1: > 75\%, 2: 10\%-75\%, 3: $\leq 10 \%)$, nuclear pleomorphism (1: uniform, 2: moderate variation in shape and size, 3 : marked variation), and mitotic count (1: 0-9/10 high-power fields [hpf], 2: 10-19/10 hpf, 3: $\geq 20 / 10 \mathrm{hpf}$ ). The sub-scores were added up to yield a total score. A total score of 3-5 corresponds to grade 1 , a total score of 6-7 corresponds to grade 2 , and a total score of 8-9 corresponds to grade 3 . The local invasiveness of the primary mouse tumors was assessed on H\&E-stained sections on the basis of their infiltration into the surrounding connective tissue. Each tumor was judged to be of "low" or "high" invasiveness, with low invasiveness being defined as a tumor that does not show any singlecell infiltrations into the surrounding tissue and high invasiveness being defined as a tumor with single-cell infiltrations into the surrounding tissue. Immunohistochemistry was carried out on paraffin-embedded sections using standard reagents and protocols (Vector Laboratories). The phospho-ErbB-2 score was analyzed analogously to the well-established ErbB-2 score: score 0 indicates absence of significant circumferential membranous staining, whereas scores $1+, 2+$, and $3+$ correspond to positive circumferential membranous staining for phospho-ErbB-2(Y1248) (1+: weak staining of $\geq 1 \%$ or moderate staining of $<10 \%$ of tumor cells; $2+$ : moderate staining of $\geq 10 \%$ or strong staining of $<30 \%$ of tumor cells; $3+$ : strong staining of $>30 \%$ of tumor cells). To test for the specificity of the goat polyclonal anti-Plexin-B1 antibody (R\&D Systems), we preincubated the antibody with the peptide used for immunization at a mass ratio of $1: 5$ for 1 hour at room temperature prior to application to the tissue slides.

Analysis of vascularization, macrophage infiltration, and apoptosis. For the anal$y$ sis of vascularization, tumor sections were stained for CD31 and imaged (3 randomly chosen fields per tumor, $\times 100$ magnification). Quantification was performed by computer-assisted digital image analysis as described previously (49). Macrophages were stained on tumor sections by an antiMac-3 antibody, and macrophages were counted by computer-assisted digital image analysis in 3 randomly chosen fields per tumor (×200 magnification). For the analysis of apoptosis, sections were stained for cleaved caspase-3, and positive cells were counted in 10 randomly selected fields per tumor ( $\times 400$ magnification).

Patients. Frozen and paraffin-embedded breast cancer tissues were provided by the tissue bank of the National Centre for Tumor Diseases (NCT, Heidelberg, Germany) (Supplemental Table 1). The ErbB-2 score was determined by immunohistochemistry using an anti-ErbB-2 antibody (Dako, clone A0485). The ErbB-2 score 0 indicates the absence of staining, whereas ErbB-2 scores $1+, 2+$, and $3+$ correspond to positive staining for ErbB-2 (1+: weak staining of $10 \%$ of tumor cells; $2+$ : moderate staining of $10 \%-30 \%$ of tumor cells; $3+$ : strong staining of $>30 \%$ of tumor cells).

Microarray analysis. The following datasets of human breast cancer patients were downloaded from the Gene Expression Omnibus (GEO) repository (http://www.ncbi.nlm.nih.gov/geo/): GSE1456, GSE2034, GSE3494, GSE4922, GSE5327, GSE7390, GSE11121, and GSE12093. All datasets were filtered for platform HG-U133A CEL files. The whole batch consisted of 1,548 arrays and was preprocessed by the Affymetrix power 
tools (normalization method, robust multiarray averaging [RMA]). The HG-U133A annotation file was downloaded from Affymetrix Annotation Files (http://www.affymetrix.com). The ERBB2 probeset ID 216836_s_at was used to group the arrays into ErbB-2-overexpressing and ErbB-2-nonoverexpressing tumors. We identified 200 arrays as ErbB-2-overexpressing and 1,348 as ErbB-2-non-overexpressing tumors. Previous studies have shown that ERBB2 mRNA expression levels correlate with protein expression levels as determined by immunohistochemistry (50). Within the group of arrays with ErbB-2 overexpression, two subgroups were defined on the basis of Plexin-B1 expression levels (215807_s_at probeset): one subgroup comprised the 60 arrays with the lowest Plexin-B1 expression, the other subgroup comprised the 60 arrays with the highest Plexin-B1 expression. The 60 arrays with low Plexin-B1 expression were mapped to the available 22 clinical datasets, and the 60 arrays with high Plexin-B1 expression were mapped to the available 39 clinical datasets. Analogously, within the group of arrays without ErbB-2 overexpression, two subgroups were defined on the basis of Plexin-B1 expression levels: one subgroup comprised the 100 arrays with the lowest Plexin-B1 expression, the other subgroup comprised the 100 arrays with the highest Plexin-B1 expression. The 100 arrays with low Plexin-B1 expression were mapped to the available 19 clinical datasets, and the 100 arrays with high Plexin-B1 expression were mapped to the available 62 clinical datasets. As the different arrays provided different clinical end points, we combined RFS (relapse-free survival), DMFS (distant metastasis-free survival), and DFS (disease-free survival) to yield one united clinical endpoint designated disease-free survival. Using survival in years and survival event data, we performed a survival analysis. Since we had sufficient events in both groups, we assumed a $\chi^{2}$ distribution with one degree of freedom. By plotting Kaplan-Meier curves using R functions (Surv and survfit), we were able to demonstrate varying survival lines. As the lines did not cross each other, we assumed the event rates to be proportional to the Cox model. Therefore, we chose the log-rank test (higher power than Wilcoxon in this setting) to compare the survival curves. The log-rank test was performed by the R survdiff function. For the analysis of overall survival, we took array groups identical to those mentioned above (ErbB-2 overexpression, 60 arrays with lowest Plexin-B1 expression, 60 arrays with highest Plexin-B1 expression) and mapped these groups to available disease-specific survival (DSS) data from the GEO datasets. We were able to map 7 arrays of the group with low Plexin-B1 expression and 13 arrays of the group with high Plexin-B1 expression to DSS values. Kaplan-Meier curves were plotted by R functions as described above. Within the group of 200 arrays with ErbB-2 overexpression, data on ER status was available for 77 arrays. Of those, 29 arrays were ER negative ( 28 with clinical outcome data) and 48 arrays were ER-positive (31 with clinical outcome data). Arrays with clinical outcome data available were sorted for Plexin-B1 expression, and Kaplan-Meier curves were plotted by R functions as described above.

Statistics. The statistical significance was evaluated by 2-tailed $t$ test (Figure 2, E, F, H, K, and L, Figure 3, B, F, and G, Supplemental Figure 2, B-D, Supplemental Figure 3C), Fisher's exact test (Figure 3D), and log-rank test (Figure 3A, Figure 4, D and E, Supplemental Figure 5, A-C). A $P$ value less than 0.05 was considered significant.

Study approval. All procedures of animal care and use in this study were approved by the local animal ethics committee (Regierungspräsidium Karlsruhe, Germany). Frozen and paraffin-embedded breast cancer tissues were provided by the tissue bank of the NCT in accordance with the regulations of the tissue bank and the approval of the ethics committee of the University of Heidelberg. Patients provided informed consent for the use of tissue.

\section{Acknowledgments}

We thank Daniel Heil and Dagmar Magalei for technical assistance. We are grateful to Hanswalter Zentgraf (Deutsches Krebsforschungszentrum [DKFZ], Heidelberg, Germany) for cooperation on the production of anti-Plexin-B1 antibodies and to Esther Herpel and the tissue bank of the NCT for providing human breast carcinoma samples. This work was supported by grants from the German Research Foundation to S. Offermanns.

Received for publication August 18, 2011, and accepted in revised form January 18, 2012.

Address correspondence to: Thomas Worzfeld or Stefan Offermanns, Max-Planck-Institute for Heart and Lung Research, Ludwigstr. 43, 61231 Bad Nauheim, Germany. Phone: 49.6032.705.1213; Fax: 49.6032.705.1204; E-mail: thomas.worzfeld@mpi-bn.mpg.de (T. Worzfeld). Phone: 49.6032.705.1201; Fax: 49.6032.705.1204; E-mail: stefan.offermanns@mpi-bn.mpg.de (S. Offermanns).
1. Slamon DJ, Clark GM, Wong SG, Levin WJ, Ullrich A, McGuire WL. Human breast cancer: correlation of relapse and survival with amplification of the HER2/neu oncogene. Science. 1987;235(4785):177-182.

2. Slamon DJ, et al. Studies of the HER-2/neu protooncogene in human breast and ovarian cancer. Science. 1989;244(4905):707-712.

3. Feigin ME, Muthuswamy SK. ErbB receptors and cell polarity: new pathways and paradigms for understanding cell migration and invasion. Exp Cell Res. 2009;315(4):707-716.

4. Hynes NE, MacDonald G. ErbB receptors and signaling pathways in cancer. Curr Opin Cell Biol. 2009;21(2):177-184.

5. Ursini-Siegel J, Schade B, Cardiff RD, Muller WJ. Insights from transgenic mouse models of ERBB2-induced breast cancer. Nat Rev Cancer. 2007;7(5):389-397.

6. Brantley-Sieders DM, et al. The receptor tyrosine kinase EphA2 promotes mammary adenocarcinoma tumorigenesis and metastatic progression in mice by amplifying ErbB2 signaling. J Clin Invest. 2008;118(1):64-78.

7. Guo W, et al. Beta 4 integrin amplifies ErbB2 signaling to promote mammary tumorigenesis. Cell. 2006;126(3):489-502.

8. Bentires-Alj M, et al. A role for the scaffold- ing adapter GAB2 in breast cancer. Nat Med. 2006;12(1):114-121.

9. Ju X, et al. Akt1 governs breast cancer progression in vivo. Proc Natl Acad Sci U S A. 2007; 104(18):7438-7443.

10. Julien SG, et al. Protein tyrosine phosphatase $1 B$ deficiency or inhibition delays ErbB2-induced mammary tumorigenesis and protects from lung metastasis. Nat Genet. 2007;39(3):338-346.

11. Schade B, et al. Distinct ErbB-2 coupled signaling pathways promote mammary tumors with unique pathologic and transcriptional profiles. Cancer Res. 2007;67(16):7579-7588.

12. Seton-Rogers SE, et al. Cooperation of the ErbB2 receptor and transforming growth factor beta in induction of migration and invasion in mammary epithelial cells. Proc Natl Acad Sci U S A. 2004;101(5):1257-1262.

13. Tamagnone L, et al. Plexins are a large family of receptors for transmembrane, secreted, and GPI-anchored semaphorins in vertebrates. Cell. 1999;99(1):71-80.

14. Neufeld G, Kessler O. The semaphorins: versatile regulators of tumour progression and tumour angiogenesis. Nat Rev Cancer. 2008;8(8):632-645.

15. Suzuki K, Kumanogoh A, Kikutani H. Semaphorins and their receptors in immune cell interac- tions. Nat Immunol. 2008;9(1):17-23.

16. Tran TS, Kolodkin AL, Bharadwaj R. Semaphorin regulation of cellular morphology. Annu Rev Cell Dev Biol. 2007;23:263-292.

17. Swiercz JM, Kuner R, Offermanns S. Plexin-B1/ RhoGEF-mediated RhoA activation involves the receptor tyrosine kinase ErbB-2. J Cell Biol. 2004;165(6):869-880.

18. Swiercz JM, Worzfeld T, Offermanns S. Semaphorin 4D signaling requires the recruitment of phospholipase $\mathrm{C}$ gamma into the plexin-B1 receptor complex. Mol Cell Biol. 2009;29(23):6321-6334.

19. Swiercz JM, Worzfeld T, Offermanns S. ErbB-2 and met reciprocally regulate cellular signaling via plexin-B1. J Biol Chem. 2008;283(4):1893-1901.

20. Aurandt J, Vikis HG, Gutkind JS, Ahn N, Guan KL. The semaphorin receptor plexin-B1 signals through a direct interaction with the Rho-specific nucleotide exchange factor, LARG. Proc Natl Acad Sci U S A. 2002;99(19):12085-12090.

21. Perrot V, Vazquez-Prado J, Gutkind JS. Plexin $B$ regulates Rho through the guanine nucleotide exchange factors leukemia-associated Rho GEF (LARG) and PDZ-RhoGEF. J Biol Chem. 2002;277(45):43115-43120.

22. Swiercz JM, Kuner R, Behrens J, Offermanns S. Plexin-B1 directly interacts with PDZ-RhoGEF/ 
LARG to regulate RhoA and growth cone morphology. Neuron. 2002;35(1):51-63.

23. Clark EA, Golub TR, Lander ES, Hynes RO. Genomic analysis of metastasis reveals an essential role for RhoC. Nature. 2000;406(6795):532-535.

24. Hall A. The cytoskeleton and cancer. Cancer Metastasis Rev. 2009;28(1-2):5-14.

25. Sahai E, Marshall CJ. RHO-GTPases and cancer. Nat Rev Cancer. 2002;2(2):133-142.

26. Vega FM, Ridley AJ. Rho GTPases in cancer cell biology. FEBS Lett. 2008;582(14):2093-2101.

27. Simpson KJ, Dugan AS, Mercurio AM. Functional analysis of the contribution of RhoA and RhoC GTPases to invasive breast carcinoma. Cancer Res. 2004;64(23):8694-8701.

28. Tang Y, Olufemi L, Wang MT, Nie D. Role of Rho GTPases in breast cancer. Front Biosci. 2008; 13:759-776.

29. Oinuma I, Ishikawa Y, Katoh H, Negishi M. The Semaphorin 4D receptor Plexin-B1 is a GTPase activating protein for R-Ras. Science. 2004;305(5685):862-865

30. Prendergast GC. Actin' up: RhoB in cancer and apoptosis. Nat Rev Cancer. 2001;1(2):162-168.

31. Deng S, et al. Plexin-B2, but not Plexin-B1, critically modulates neuronal migration and patterning of the developing nervous system in vivo. J Neurosci. 2007;27(23):6333-6347.

32. Giacobini P, et al. Semaphorin $4 \mathrm{D}$ regulates gonadotropin hormone-releasing hormone-1 neuronal migration through PlexinB1-Met complex. J Cell
Biol. 2008;183(3):555-566.

33. Rody A, et al. Poor outcome in estrogen receptorpositive breast cancers predicted by loss of plexin B1. Clin Cancer Res. 2007;13(4):1115-1122.

34. Fazzari $\mathrm{P}$, et al. Plexin-B1 plays a redundant role during mouse development and in tumour angiogenesis. BMC Dev Biol. 2007;7:55

35. Ye S, et al. Plexin-B1 silencing inhibits ovarian cancer cell migration and invasion. BMC Cancer. 2010;10:611.

36. Argast GM, et al. Plexin B1 is repressed by oncogenic B-Raf signaling and functions as a tumor suppressor in melanoma cells. Oncogene. 2009; 28(30):2697-2709.

37. Gomez Roman JJ, et al. Plexin B1 is downregulated in renal cell carcinomas and modulates cell growth. Transl Res. 2008;151(3):134-140.

38. Qiang R, et al. Plexin-B1 is a target of miR-214 in cervical cancer and promotes the growth and invasion of HeLa cells. Int J Biochem Cell Biol. 2011;43(4):632-641.

39. Barberis D, et al. p190 Rho-GTPase activating protein associates with plexins and it is required for semaphorin signalling. J Cell Sci. 2005;118(pt 20):4689-4700.

40. Giordano S, et al. The semaphorin $4 \mathrm{D}$ receptor controls invasive growth by coupling with Met. Nat Cell Biol. 2002;4(9):720-724.

41. Franco M, Tamagnone L. Tyrosine phosphorylation in semaphorin signalling: shifting into overdrive. EMBO Rep. 2008;9(9):865-871.

42. Hudis CA. Trastuzumab - mechanism of action and use in clinical practice. N Engl J Med. 2007; 357(1):39-51.

43. Jones KL, Buzdar AU. Evolving novel anti-HER2 strategies. Lancet Oncol. 2009;10(12):1179-1187.

44. Lan KH, Lu CH, Yu D. Mechanisms of trastuzumab resistance and their clinical implications. Ann NY Acad Sci. 2005;1059:70-75.

45. Crone SA, et al. ErbB2 is essential in the prevention of dilated cardiomyopathy. Nat Med. 2002; $8(5): 459-465$.

46. Ozcelik C, et al. Conditional mutation of the ErbB2 (HER2) receptor in cardiomyocytes leads to dilated cardiomyopathy. Proc Natl Acad Sci U S A. 2002;99(13):8880-8885

47. Köhler G, Milstein C. Continuous cultures of fused cells secreting antibody of predefined specificity. Nature. 1975;256(5517):495-497.

48. Hill CS, Wynne J, Treisman R. The Rho family GTPases RhoA, Rac1, and CDC42Hs regulate transcriptional activation by SRF. Cell. 1995;81(7):1159-1170.

49. Wild R, Ramakrishnan S, Sedgewick J, Griffioen AW. Quantitative assessment of angiogenesis and tumor vessel architecture by computer-assisted digital image analysis: effects of VEGF-toxin conjugate on tumor microvessel density. Microvasc Res. 2000;59(3):368-376.

50. Press MF, et al. HER-2 gene amplification, HER-2 and epidermal growth factor receptor mRNA and protein expression, and lapatinib efficacy in women with metastatic breast cancer. Clin Cancer Res. 2008;14(23):7861-7870. 